\title{
COLOURED IMAGE SEGMENTATION USING K-MEANS ALGORITHM
}

\author{
${ }^{1}$ FENWA O.D. ${ }^{2}$ ALO O.O. AND ${ }^{3}$ FALOHUN A.S. \\ ${ }^{123}$ Dept. of Computer Sc. \& Eng, LAUTECH P.M.B 4000, Ogbomoso, Oyo State, Nigeria \\ Corresponding Authors email: \{odfenwa/ ooalo/ asfalohun\}@lautech.edu.ng
}

\begin{abstract}
Very large collections of images are growing ever more common. From stock photo collections to proprietary databases to the Web, these collections are diverse and often poorly indexed; unfortunately, image retrieval systems have not kept pace with the collections they are searching. The shortcomings of these systems are due both to the image representations they use and to their methods of accessing those representations to find images: (i) When users generally want to find images containing particular objects, most existing image retrieval systems represent images based only on their low-level features ("stuff") with little regard for the spatial organization of those features. (ii) Systems based on user querying are often unintuitive and offer little help in understanding why certain images were returned and how to refine the query. Often the user knows only that he has submitted a query for, say, a bear and retrieved very few pictures of bears in return. (iii) For general image collections, there are currently no systems that automatically classify images or recognize the objects they contain. Hence, in this paper K-means algorithm was used to segment colors in two common skin diseases; Ringworm and Scabies. It was noted that K-means result generates as much clusters based on colors in the image, the k-means result can also extend as far as ten colors, if these colors are available on the image, and they generate subsequent coloration. The result gotten was further used against the more popular Fuzzy Cluster Means (FCM) and the results showed that K-Means outperform the Fuzzy Cluster Means because FCM could not generate enough segmented regions because it works majorly on grayscale (black and white) images and has little performance in comparison to the K-means algorithm. The results obtained also showed other identifiable region which are needed for proper diagnosis by the consulting physician. However, Scabies skin diseases could not yield more and accurate results due to poor lighting effect of the image. This shows that light plays a major role in the segmentation of images based on color.
\end{abstract}

\section{Keywords}

Image Segmentation, Color quantization, Skin Disease, Ringworm, K-means, Clusters, Scabies.

\section{Academic Discipline and Sub-Disciplines}

Computer Science, Computer Engineering, Information Technology, Information Systems

\section{Subject Classification}

Pattern Recognition, Artificial Intelligence, Machine Learning

\section{TYPE (METHOD/APPROACH)}

Experimentation, Empirical studies, Computer Simulation

\section{Council for Innovative Research}

\author{
Peer Review Research Publishing System
}

\section{Journal: INTERNATIONAL JOURNAL OF COMPUTERS \& TECHNOLOGY}

Vol. 15 , No. 3

www.ijctonline.com, editorijctonline@gmail.com 


\section{INTRODUCTION}

One major task of pattern recognition, image processing and related areas is to segment image into homogenous regions. Image segmentation is the first step towards image understanding and image analysis. Image segmentation has been acknowledged to be one of the most difficult tasks in computer vision and image processing ([1]; [2]; [3]). Unlike other vision tasks such as parametric model estimation [4], fundamental matrix estimation, optical flow calculation, etc., there is no widely accepted model or analytical solution for image segmentation. There probably is no "one true" segmentation acceptable to all different people and under different psychophysical conditions.

Color image segmentation is a process of extracting from the image domain one or more connected regions satisfying uniformity (homogeneity) criterion which is based on feature(s) derived from spectral components. These components are denned in a chosen color space model. The segmentation process could be augmented by some additional knowledge about the objects in the scene such as geometric and optical properties. Color is a very important cue in extracting information from images [5]. Color histograms are commonly used in content-based retrieval systems and have proven to be very useful, however, the global characterization is poor at for example, distinguishing between a field of orange flowers and a tiger, because it lacks information about how the color is distributed spatially. It is important to group color in localized regions and to fuse color with textural properties [6].

The importance of skin diseases is often overlooked. They are usually not life-threatening and tend to be "shrugged away". Skin diseases are however a significant problem all over the world. Nowadays many skin diseases are related to or influenced by concomitant HIV infection. These conditions are presented rather than other rare skin diseases. There are many more congenital malformations and syndromes, benign and malignant tumors, tropical infections, skin manifestations of systemic and metabolic disease, auto-immune diseases etc. which are important but are not common. When care is taken to make the proper diagnosis and to institute the proper treatment the management of skin diseases often results in great improvement and satisfaction for patient and health care worker alike. Treatments required generally need not be expensive and are locally available [7].

\section{METHODOLOGY}

This paper focuses on a broad domain of photographic images of the skin diseases: Ringworm and Scabies. A challenging aspect of this work is that we attempt to accomplish both feature extraction and segmentation with relatively low resolution and occasionally degraded or compressed images, just as humans do. This is especially important since low resolution images are most frequently used within WWW documents. In addition, the advantage of low resolution images is that access and processing time are significantly reduced. Color of an image can carry much more information than gray level. There probably is no "one true" segmentation acceptable to all different people and under different psychophysical conditions. Researchers have extensively worked over this fundamental problem and proposed various methods for image segmentation. These methods can be broadly classified into seven groups: (1) Histogram thresholding, (2) Clustering (Fuzzy and Hard), (3) Region growing, region splitting and merging, (4) Edge-based, (5) Physical modelbased, (6) Fuzzy approaches, and (7) Neural network and GA (Genetic algorithm) based approaches [8]. Clustering (Fuzzy) method is adopted in this paper. However, different types of segmentation techniques exist in the process of segmenting images. These techniques include Pixel Based Segmentation, Region Based Segmentation, Edge Based Segmentation and Physics Based Segmentation. Pixel based technique is adopted in this paper. The model architecture showing the stages of the system development is as shown in figure 2.1 

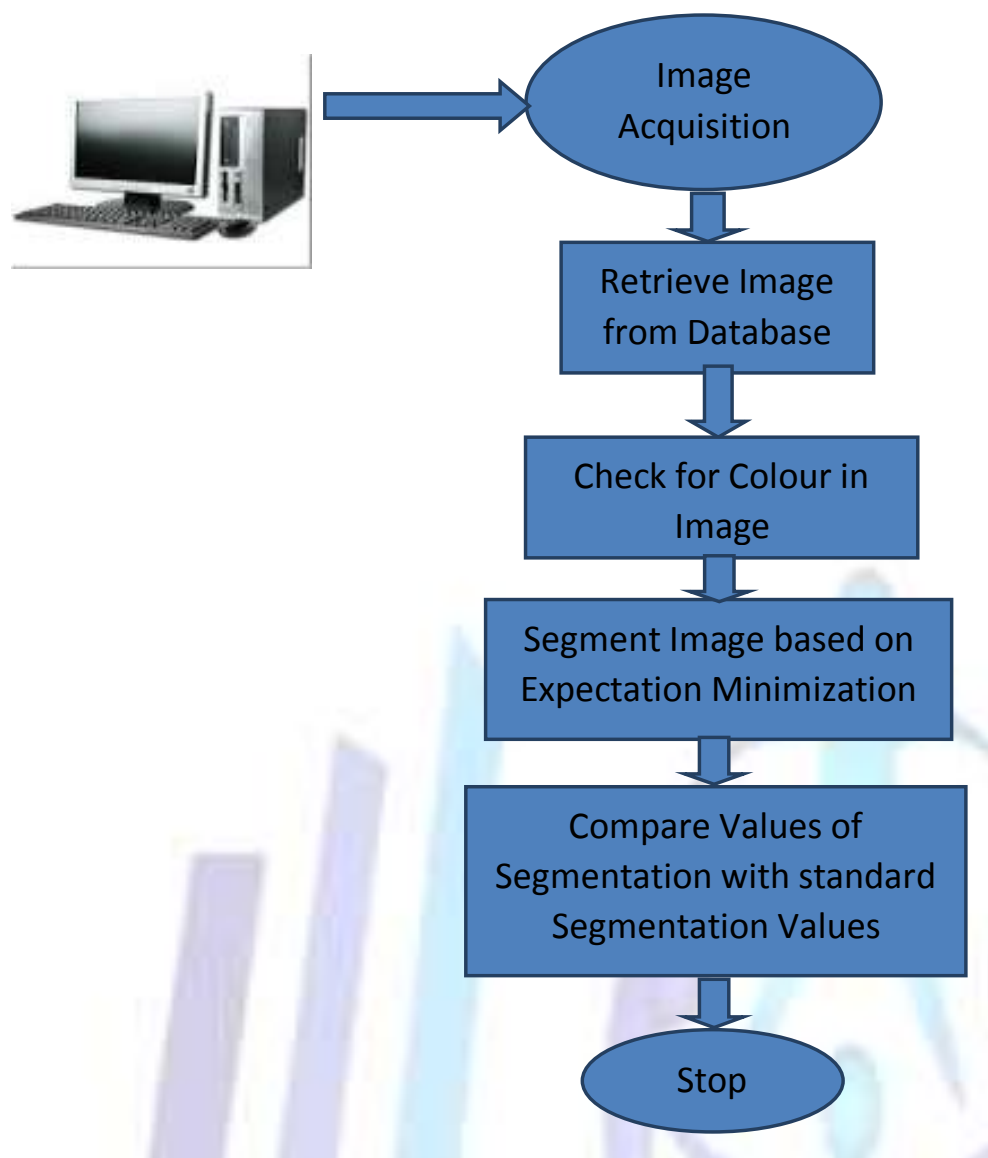

Figure 2.1 Model Architecture of the Developed System

\subsection{Image Acquisition}

A very large database is required to collect images and store them. The database would contain images from different views which include photographic images which could either be from landscape, cityscapes, paths, plants, animals, objects, and other multimedia items. As soon as these images are acquired and collected they are stored in the database in which they are called from.

\subsection{Pre-processing}

Pre-processing of image is necessary before any image analysis can be carried out. It involves filtering, selecting, randomizing, conversion to gray-scale, resizing and removal of objects that could affect the proper processing of the images. Before a computer vision method can be applied to image data in order to extract some specific piece of information, it is usually necessary to process the data in order to ensure that it satisfies certain assumptions implied by the method.

\subsection{Image Retrieval by Querying}

Anyone who has used a search engine, text-based or otherwise, is familiar with the reality of unwanted matches. Often in the case of text searches this results from the use of ambiguous keywords, such as "bank" or "interest". Unfortunately, with image queries it is not always so clear why things go wrong. Unlike with text searches, in which the user can see the features (words) in a document, none of the current content-based image retrieval systems allows the user to see exactly what the system is looking for in response to a similarity-based query. Simply allowing the user to submit an arbitrary image (or sketch) and set some abstract knobs without knowing how they relate to the input image in particular implies a degree of complexity that searching algorithms do not have. As a result, a query for a bear can return just about any object under the sun if the query is not based on image regions, the segmentation routine fails to "find" the bear in the submitted image, or the submitted image contains other distinctive objects. Without realizing that the input image was not properly processed, the user can only wonder what went wrong. In order to help the user formulate effective queries and understand their results as well as to minimize disappointment due to overly optimistic expectations of the system, the system should display its representation of the submitted image and the returned images. 


\subsection{Segmentation Based on Expectation-Minimization}

Once an image has been processed using selected feature extraction scheme, the result is a large set of $6-D$ feature vectors, which we may regard as points in a 6-D feature space. In order to divide these points into groups, we make use of the Expectation-Maximization (EM) algorithm to determine the maximum likelihood parameters of a mixture of $\mathrm{K}$ Gaussians inside the 6-D feature space. The EM algorithm is used for finding maximum likelihood parameter estimates when there is missing or incomplete data. In this paper, the missing data is the region to which the points in the feature space belong. We estimate values to fill in for the incomplete data (the "E-Step"), compute the maximum likelihood parameter estimates using this data (the "M-Step"), and repeat until a suitable stopping criterion is reached.

The first step in applying the EM algorithm is to initialize a mean vector and covariance matrix to represent each of the $\mathrm{K}$ groups. We initialize the means to random values and the co-variances to identity matrices. We chose the initialization for EM carefully, but we have found that the initialization has little effect on the quality of the resulting segmentation.) The update scheme allows for full covariance matrices; variants include restricting the covariance to be diagonal or a constant times the identity matrix. Full covariance matrices suit our problem, since many plausible feature clusters require extruded covariance shapes, e.g. the shades of gray along the color cone axis. Upon convergence, the Gaussian mixture parameters can be inspected to determine what color/texture properties are represented by each component of the mixture. Some examples of groups that can form include the following: bright, bluish and texture less regions (e.g., sky) anisotropic and non-polar regions, green weak-isotropic texture (e.g., grass)

Ideally we have chosen the value of $\mathrm{K}$ that best suits the natural number of groups present in the image. One readily available notion of goodness of fit is the log-likelihood. Given this indicator, we apply the Minimum Description Length (MDL) principle to select among values of $\mathrm{K}$. As a consequence of this principle, when models using two values of $\mathrm{K}$ fit the data equally well, the simpler model is chosen. For our experiments, $\mathrm{K}$ ranges from 2 to 5 . Once a model is selected, the next step is to perform spatial grouping of those pixels belonging to the same color/texture cluster. We first produce a K-level image which encodes pixel-cluster memberships by replacing each pixel with the label of the cluster for which it attains the highest likelihood. To enforce a small amount of spatial smoothness in this representation, we apply a $3 \times 3$ maximum-vote filter to the raw cluster-membership image. Finally, we run the resulting image through a connected components algorithm to produce a set of labeled image regions (Alternatively, one could enforce spatial constraints by appending the pixel coordinates to the feature vectors, though we observed that this method too often yields unsatisfactory segmentations)

\section{ALGORITHM FOR K-MEANS}

K-Means is a rather simple but well known algorithm for grouping objects, clustering. The K-Means method is numerical, unsupervised, non-deterministic and iterative Again all objects need to be represented as a set of numerical features. In addition the user has to specify the number of groups (referred to as k) he wishes to identify. Each object can be thought of as being represented by some feature vector in an $n$ dimensional space, $n$ being the number of all features used to describe the objects to cluster. The algorithm then randomly chooses $\mathrm{k}$ points in that vector space, these points serve as the initial centres of the clusters. Afterwards all objects are each assigned to centre they are closest to. Usually the distance measure is chosen by the user and determined by the learning task. After that, for each cluster a new centre is computed by averaging the feature vectors of all objects assigned to it. The process of assigning objects and recomputing centers is repeated until the process converges. The algorithm can be proven to converge after a finite number of iterations. Several tweaks concerning distance measure, initial centre choice and computation of new average centers have been explored, as well as the estimation of the number of clusters $\mathrm{k}$. Yet the main principle always remains the same do that for you.

\section{The K-means Algorithm}

Step 1: Load Image

Step 2: Read Image into Matlab

Step 3: Convert Image from RGB Color Space to L*a*b* Color Space

Step 4: Classify the colors in $a^{*} b$ spacing using K-means segmentation

Step 5: Label every pixel in the image using the results from K-means

Step 6: Create Images that segment the $\mathrm{H}$ and $\mathrm{E}$ image by color

Step 7: Segment the nuclei into a separate image

Step 8: Stop.

\section{RESULTS AND DISCUSSION}

The system user interface was designed and developed on Matlab 7.10a and computed on a 4-GHZ memory. The user interface is flexible as it loads the image onto it and also gives out results. The results are displayed for visibility and further studies. 


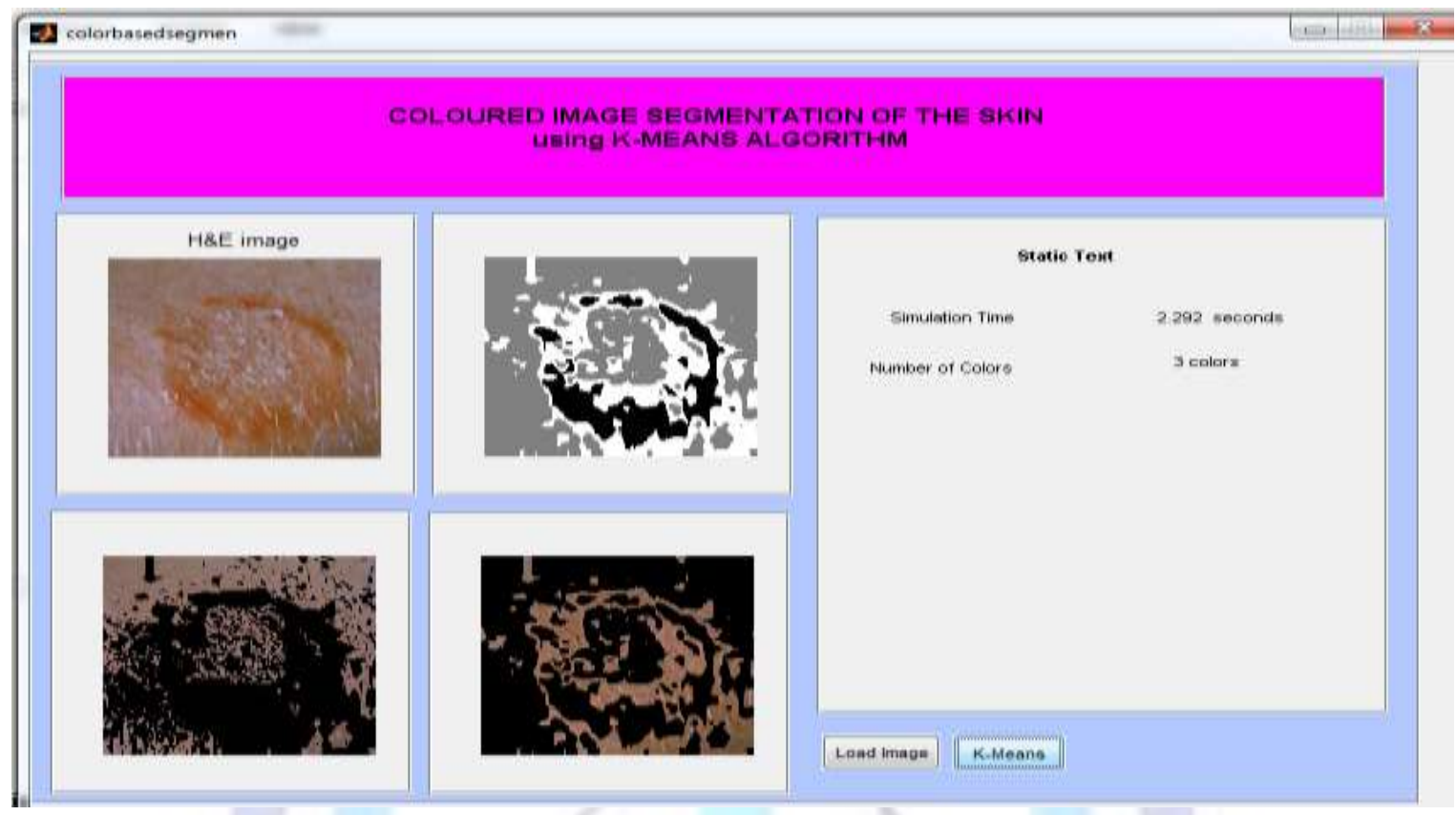

Figure 4.1 System Design Interface

Figure 4.1 above shows the system design interface used for loading the images into the system and selecting the Kmeans button. The k-means segment this image based on the number of colors found in the image. The segmentation results show other hidden part of the image which is not visible under microscope or other magnifying tools. Each color in the image represents a distinct feature found in the image. In figure 4.2 below, the original image is shown with brown skin coloration. The disease associated with the skin is Ringworm, which is a concentric circle centered round the skin.

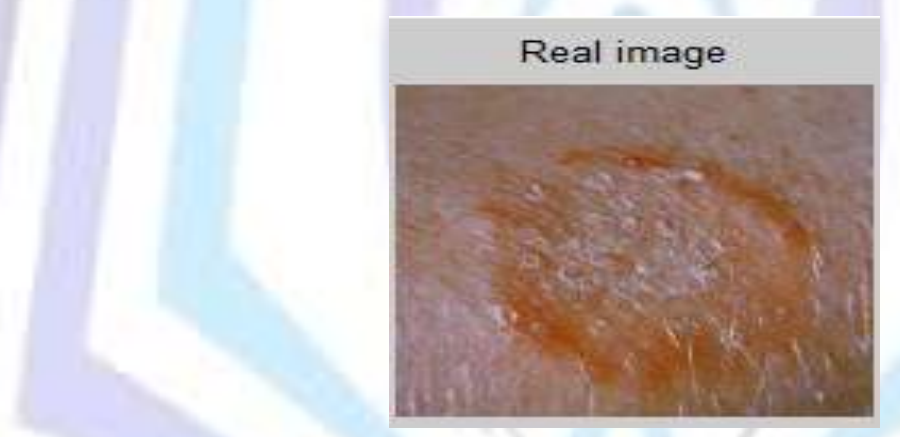

Figure 4.2 Skin with Ringworm infection

(Source: http://f.tqn.com/y/dermatology/1/9/V/8/Rigworm.jpg)

First, colors in the image are coarsely quantized without significantly degrading the color quality. The purpose is to extract a few representing colors that can be used to differentiate neighboring regions in the image. Typically, 10-20 colors are needed in the images of natural scenes. A good color quantization is important to the segmentation process. A perceptual color quantization algorithm is used in our implementation.

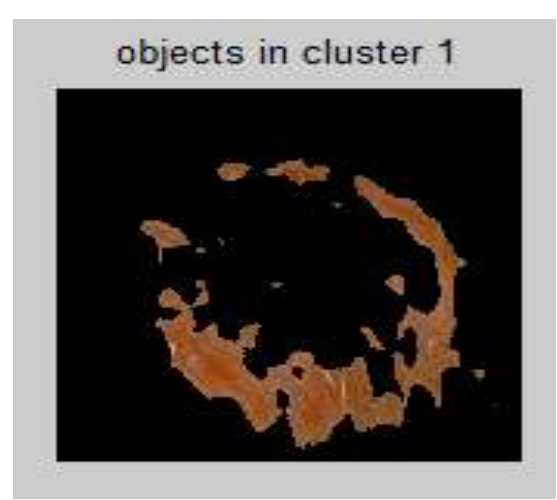

Figure 4.3 Objects in cluster 1

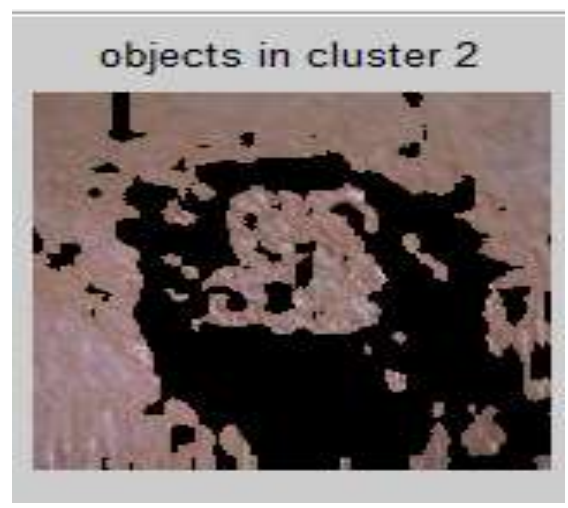

Figure 4.4 Objects in cluster 2 
Clusters are used to separate distinguishing feature of the image based on color or frequency of the color. To the physician, this might represent new information needed to base his diagnostic reasoning. For the object in cluster 1 , threshold of the color identifies structures that are not identifiable. This is in line with the clustering algorithm of the $\mathrm{k}$ means. As observed in figure 4.4, the image highlights some features different from the figure 4.3, for the physician, more ring worm appearance are located in the skin, which can be easily seen and quickly treated. The spread of the ringworm to other part of the body could be easily noticeable with the aid of the color segmentation, but with the normal eye it is not really feasible.

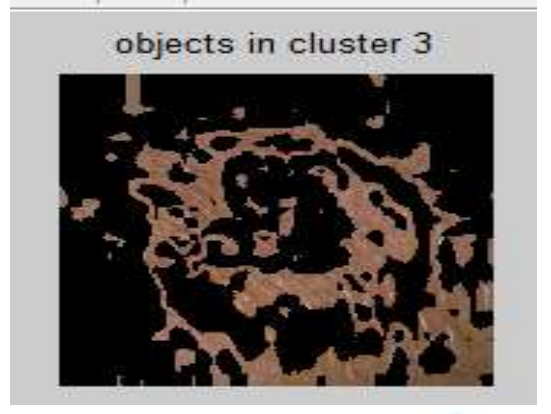

Figure 4.5 Objects in cluster 3

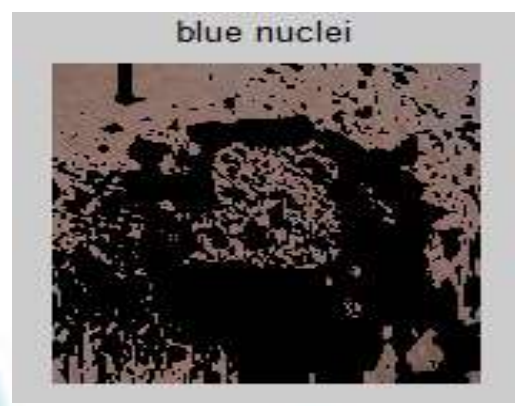

Figure 4.6 Blue nuclei of image

As shown in figure 4.5 above, more ringworm-like structure starts to appear in the surface, the more the color, the more the distinguishing features. The ringworm appearance in other parts of the skin shows the easy transmission of the disease to the other parts of the skin. Some color quantization may appear dim as it may and may require further segmentation process. The major criterion behind proper segmentation is the lighting effect the image has i.e. the amount of light the image was exposed to while being captured. The image shown figure 4.6 above is the blue nuclei of the image identified, and its subsequent result. The blue nuclei is used to bring out more hidden features which are not retrieved by clusters 1, 2 and 3. The blue nuclei are also used to point out those features for easily identification of skin diseases.

\subsection{Simulation Time}

Simulation time for the process is a bit faster and shorter with respect to the memory of the system, the simulation time is important as it helps dictate how fast or slow the process was.

\subsection{K-means Result as Compared to Fuzzy Cluster Means (FCM)}

The k-means result generates as much clusters based on colors in the image, the k-means result can extends as far as ten colors, if these colors are available on the image, and they generate subsequent coloration. As for the Fuzzy Cluster Means (FCM), it works majorly on grayscale (black and white) images, and has little performance in comparison to the kmeans algorithm.

\subsection{Results for the Skin Disease (Scabies)}

The scabies skin disease is a disease which involve itching and smaller red dots on the body of the skin, as shown in the figure 4.6 below.

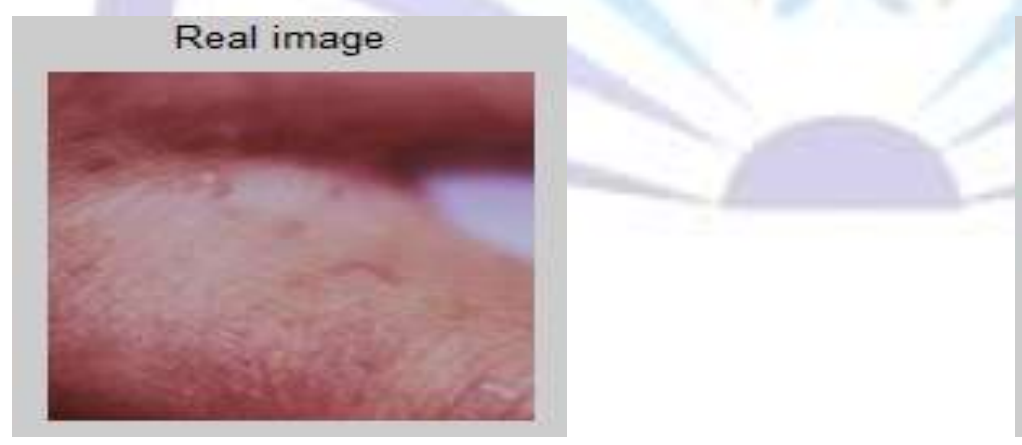

Figure 4.6 Scabies Skin Disease

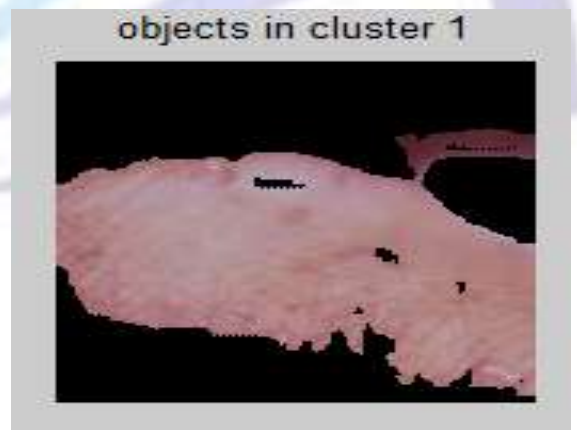

Figure 4.7 Objects in cluster 1

(Source: http://f.tan.com/y/dermatology/1/9/9/6/scabies_web.jpg)

Clusters are used to separate distinguishing features of the image based on color or frequency of the color. To the physician, this might represent new information needed to base his diagnostic reasoning. Cluster 1 threshold of the color identifies structures that are not identifiable. This is in line with the clustering algorithm of the K-Means. 


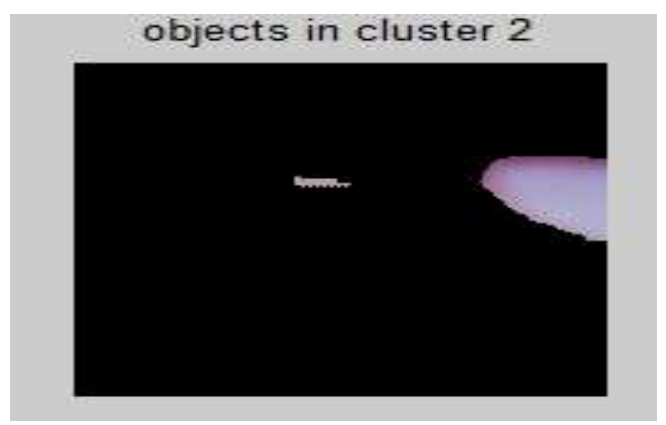

Figure 4.8 Objects in Cluster 2

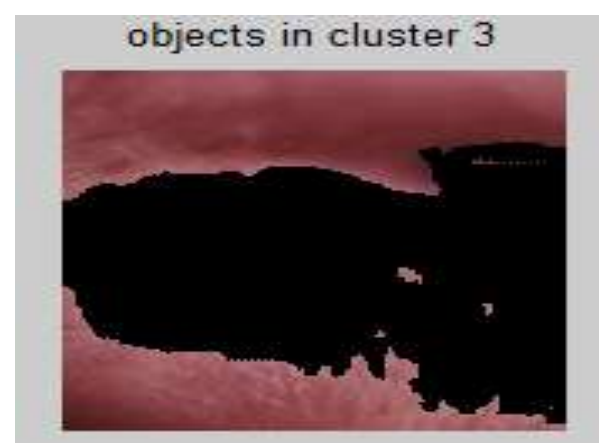

Figure 4.9 Objects in cluster 3

As observed and as different from the first image, this image highlights some features different from the figure 4.8, for the physician, for the scabies, dark areas are located in the skin which cannot be easily seen and quickly treated. The spread of the ringworm to other part of the body could be easily noticeable with the aid of the color segmentation, but in the case of the scabies it is not. The scaly looking image of the scabies disease is shown in cluster 3 in figure 4.9 , due to the coloration or lighting effect of the image, it is seen and discovered that the image itself does not seem to have enough useful information. In such cases most information are dependent on the blue nuclei.

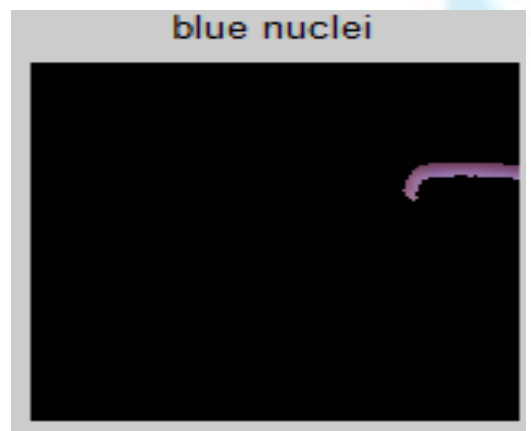

Figure 4.10 Image showing blue nuclei

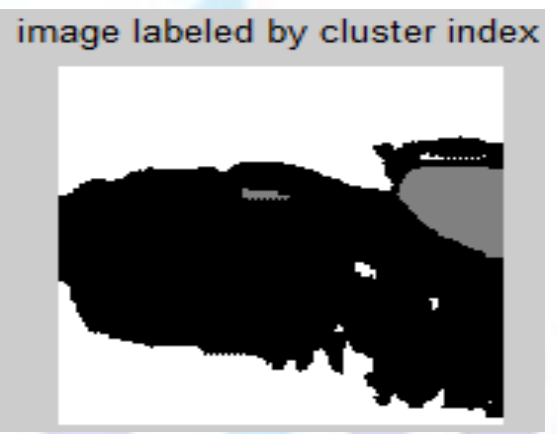

Figure 4.11 Image labeled by cluster index

The blue nuclei in figure 4.10 gives a low information about the image making it look blurred, and not direct in terms of diagnosis, the physician might have to rely on other information such as cluster index in figure 4.11 . Here, the image is seen to be visible and the parts with scabies infection are shown deep and clearly as distinguished from the original image.

\subsection{Reason for Poor Performance for Colored Image Segmentation of Scabies}

The major reason while the scabies disease image yielded poor results are due to poor lighting effect while acquiring image through digital snapshot or multimedia cameras. The intensity of light can change the way an image responds to segmentation types. Colors in image are still a sum up for light (white), i.e. light plays a major role in coloration and segmentation of the medical images.

\section{CONCLUSION AND RECOMMENDATIONS}

Color image segmentation is a process of extracting from the image domain one or more connected regions satisfying uniformity (homogeneity) criterion which is based on feature(s) derived from spectral components. These components are denned in a chosen color space model. Color is a very important clue in extracting information from images. Color histograms are commonly used in content-based retrieval systems and have proven to be very useful in extracting information from images.

In this paper, the popular K-Means algorithm was used to segment colors in two common skin diseases: Ringworm and Scabies. The results obtained showed other identifiable region which are needed for proper diagnosis by the consulting physician. It is also noteworthy to say that scabies skin diseases could not yield more and accurate results due to poor lighting effect of the image. This shows that light plays a major role in the segmentation of images based on color. The result gotten was also used against the more popular Fuzzy Cluster Means (FCM) and the results show that KMeans outperform the Fuzzy Cluster Means because FCM could not generate enough segmented regions.

From the results obtained, the following were recommended:

$>\quad$ Other skin diseases such as cancer of the skin could be segmented and the results evaluated

$>\quad$ A content-based retrieval system should be created for colored image segmentation

$>\quad$ A comparative analysis could be made between color based segmentation and edge based segmentation of medical images 
Medical practitioner should accept and imbibe telemedicine as a new technology in their field of study.

$>\quad$ Students of Higher Institution should be taught Image processing as a core course.

$>\quad$ Matlab, and other higher programming languages should be taught at lower levels, to enable students familiarize themselves with it.

\section{References}

[1] Cheng, H.D., et al., (2001). Color Image Segmentation: Advances and Prospects. Pattern Recognition. 34: p. 2259-2281

[2] Comaniciu, D. and P. Meer, (1999). Distribution Free Decomposition of Multivariate Data. Pattern Analysis and Applications. 2: p. 22-30.

[3] Wang, H. and D. Suter. A Novel Robust Method for Large Numbers of Gross Errors. In Seventh International Conference on Control, Automation, Robotics And Vision (ICARCV02). 2002. Singapore: p. 326-331.

[4] Wang, H. and Suter, D. (2003). Color Image Segmentation Using Global Information and Local Homogeneity. Proc. VIIth Digital Image Computing: Techniques and Applications, Sun C., Talbot H., Ourselin S. and Adriaansen T. (Eds.), 10-12

[5] Skarberk, W. and Koschan, A. (1994). Color Image Segmentation-A survey. An Unpublished Thesis in the Institute of Technical Information-Berlin.

[6] Belongie, S. Carson, C., Greenspan, H. and Malik, J. (1997). Color- and Texture-Based Image Segmentation Using EM and Its Application to Content-Based Image Retrieval. ICCV98.

[7] Hees Colette van and Ben Naafs (2003). Common Skin Diseases in Africa.

[8] Dutta, S. Chaudhuri, B. B. (2009). Homogenous Region based Color Image Segmentation. Proceedings of the World Conference on Engineering and Computer Science.

\section{AUTHORS' BIOGRAPHIES}

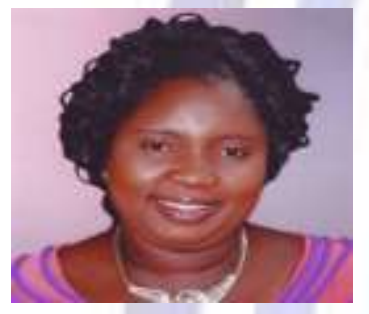

Olusayo D. FENWA is a Senior Lecturer at the Department of Computer Science and Engineering, Ladoke Akintola University of Technology, Ogbomoso, Nigeria where she teaches undergraduate and postgraduate students, supervises students' project/thesis and does research. Deborah obtained a Bachelor degree (B.Tech. with Honours) in Computer Science from Ladoke Akintola University of Technology, Ogbomoso, Oyo state, Nigeria and her Masters (MSc.) and Doctorate (PhD) degrees in Computer Science from the same university. Dr. (Mrs) Fenwa currently has several peer reviewed journal articles and conference papers across different research areas of Computer Science and Engineering including Pattern Recognition, Artificial Intelligence and Information Systems. Deborah is happily

married with kids.

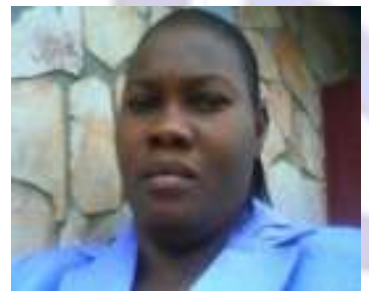

Oluwaseun O. ALO is a Lecturer at the Department of Computer Science and Engineering, Ladoke Akintola University of Technology, Ogbomoso, Nigeria where she teaches undergraduate and postgraduate students, supervises students' project/thesis and does research. Oluwaseun obtained a Bachelor degree (B.Tech. with Honours) in Computer Science from Ladoke Akintola University of Technology, Ogbomoso, Oyo state, Nigeria and her Masters (MSc.) and Doctorate (PhD) degrees in Computer Science from the same university. Dr. (Mrs) Alo currently has several peer reviewed journal articles and conference papers across different research areas of Computer Science and Engineering including Information Systems, Artificial Intelligence and Computational Complexity. She is happily married with kids.

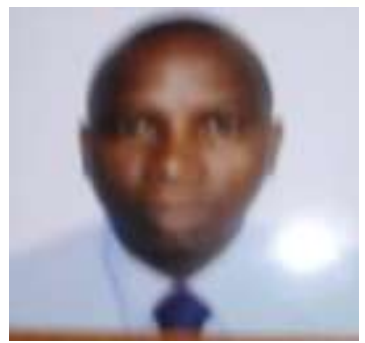

Adeleye S. FALOHUN is a Senior Lecturer at the Department of Computer Science and Engineering, Ladoke Akintola University of Technology, Ogbomoso, Nigeria where he teaches undergraduate and postgraduate students, supervises students' project/thesis and does research. He obtained a Bachelor degree (B.Tech. with Honours) in Computer Engineering from Ladoke Akintola University of Technology, Ogbomoso, Oyo state, Nigeria and his Masters (MSc.) and Doctorate (PhD) degrees in Computer Science from the same university. Dr. Falohun currently has several peer reviewed journal articles and conference papers across different research areas of Computer Science and Engineering including Pattern Recognition, Artificial Intelligence and Information Systems. Adeleye is happily married with kids. 